\title{
POUR UNE HISTOIRE CULTURELLE DU SNOBISME MUSICAL'
}

\author{
Didier Francfort \\ Institut d'Histoire Culterelle Européenne - Bronisław Geremek / Université \\ de Lorraine \\ arrivefrancfort@aol.com
}

"La chouette de Minerve ne prend son envol qu'au crépuscule », Hegel invite à penser que lorsque l'on étudie un phénomène dans les sciences humaines et sociales, il est déjà trop tard: il n'existe plus. Le colloque de Ribeirão Preto invitant à réféchir, de façon interdisciplinaire ouverte, au phénomène du snobisme s'impose d'autant plus que l'on peut se poser la question de savoir si ce «snobisme musical » existe encore, pour poser la question de son articulation avec l'idée d'avantgarde. Les sociétés contemporaines connaissent-elles encore la forme spécifique de «distinction», selon le schéma de Pierre Bourdieu, qui est considérée comme du snobisme? Là réside la première difficulté, le fait d'être snob est toujours une assignation externe jamais une identité assumée, sauf dans une chanson paradoxale de 1954, signée par Boris Vian et Jimmy Walter : "J'suis snob ». Le snob, c'est toujours d'abord l'autre. Marcel Proust fait du personnage de l'ingénieur Legrandin le prototype du snob, qui passe son temps à dénoncer le snobisme des autres : "des tirades enflammées qu'il entamait souvent contre l'aristocratie, la vie mondaine, le snobisme, «certainement le péché auquel pense saint Paul quand il parle du péché pour lequel il n'y a pas de rémission $»^{2}$. Dès la première apparition du mot, en Angleterre, les snobs sont présentés au monde par «l'un d'entre eux ». En effet, lorsqu'en 1848

- Conferencia proferida no dia 16 de outubro de 2014, no Departamento de Música da Faculdade de Filosofia, Ciências e Letras de Ribeirão Preto da Universidade de São Paulo, dentro da programação do VI Encontro de Musicologia. Disponivel em https:/l youtu.be/XvyNfvOXOCo?list=UU7kMPRd6yA9Pulnjl6voHXA (acesso: 10/1 1/20 15).

2 Marcel Proust, Du côté de chez Swann, Pléiade p. 68. 
William Makepeace Thackeray rassembla ses articles parus dans Punch, il donna au recueil le titre «The Snobs of England, By One of Themselves » («es Snobs d'Angleterre, par l'un d'entre eux »).

Nous nous proposons ici d'apporter une réflexion d'histoire culturelle sur le snobisme musical comme étant une forme particulière d'un phénomène culturel et social global. Si l'étymologie du mot snob comme étant l'abréviation qui désignait les roturiers sur les registres d'inscription des Public Schools («sine nobilitate ») est très discutée, la réalité de la démarcation entre les snobs et les nobs est très perceptible au milieu du XIXe siècle. Ce qui impliquerait que par rapport aux formes générales de distinction opposant les élites à la plèbe, on a affaire à un double rapport social de volonté de se faire passer pour ce que l'on n'est pas et de volonté de se démarquer des autres. Le phénomène implique donc qu'il y ait concurrence entre élites, entre vieux riches et parvenus, entre nobles et roturiers. On pourrait peut-être remonter à la splendeur démonstrative et à l'opulence des festins donnés par l'affranchi Trimalcion dans le Satyricon de Pétrone (puis de Fellini). Mais il ne suffit pas de donner des démonstrations d'opulence et d'être prodigue pour être snob, il faut faire preuve de raffinement, de distinction et se démarquer de ceux qui, sur le seuil de l'admission aux cercles les plus étroits des élites, n'ont pas réussi à percevoir tous les codes. La caractéristique du snobisme est la volonté et la capacité de maîtriser l'espace culturel en choisissant un terrain privilégié et unique d'affirmation de richesse culturelle personnelle. On ne se dit pas snob mais on se dit wagnérien et non mélomane. L'unicité normative est au cœur de l'énoncé snob. «II n'y a qu'une interprétation audible » d'un œuvre. Voilà le type du discours musical snob par excellence. Et c'est peut-être un certain doute sur l'unicité de l'échelle des valeurs et des esthétiques qui peut conduire à penser que le snobisme est mort.

\section{La fin du snobisme?}

Les approches historiques consistent souvent à limiter dans le 
temps ce qui est perçu comme un phénomène immémorial et immuable. Les ouvrages devenus classiques des «constructivistes » mettant en évidence le caractère récent des «traditions inventées »3. On pourrait considérer que pour que le snobisme existe il faut qu'il y ait une bourgeoisie et une aristocratie distincte. La référence demeure bien celle du Bourgeois gentilhomme de Molière. En 1896, Pierre Veber parle de « bourgeois-gentilhommes de l'esthétique $»^{4}$. Mais le début de l'opposition entre nobles et bourgeois est peut-être plus facile à situer dans la chronologie que la fin. Cette fin supposée du snobisme n'implique pas la disparition des élites par nivellement social, ni leur unification alors que l'écart entre les «classes moyennes » et les cercles étroits de la grande richesse semble s'accentuer.

Le corolaire de cette apparente dilution du snobisme est plutôt la diversification de l'échelle des valeurs et des esthétiques. La maîtrise d'un capital culturel univoque ne suffit plus. "Le parfait wagnérien » de l'époque de Bernard Shaw ne peut rivaliser avec le bourlingueur mondain capable de charmer les auditoires avec ses récits de voyages exotiques, sa connaissance de mets inconnus et de musiques inouïes. Par ailleurs, la distinction culturelle est, dans la vie mondaine, souvent marginalisée. La plupart des politiques ne lit guère. Cela n'est certainement pas nouveau. Ce qui est modifié est qu'auparavant, naguère, ils ne s'en vantaient pas. A présent, un président de la République peut affirmer qu'une fonctionnaire n'a pas besoin de connaître La Princesse de Clèves, une ministre de la Culture peut avouer ne pas connaître de titres d'un écrivain qui vient de recevoir le Prix Nobel de Littérature. De façon générale, la communication politique implique de façon continue une forme d'antisnobisme qui consiste à montrer que l'homme politique n'est pas élitiste qu'il peut être proche des pratiques culturelles populaires et non mondaines. Pour nous en tenir à la musique et aux pratiques musicales, la recherche de la légitimité culturelle ne conduit plus les chefs d'Etat et les hommes de gouvernement à montrer leur goût pour une

\footnotetext{
3 Eric Hobsbawm E Terence Ranger (orgs.). A invenção das tradições. - Rio de Janeiro: Paz e Terra, 1984.

${ }^{4}$ Pierre Veber, Chez les snobs, roman. 2e édition P. Ollendorff, 1896, 296 p.
} 
musique classique ou contemporaine mais à apparaître comme étant proche des musiques populaires, en assumant un positionnement paradoxal. On est passé, dans la sphère publique symbolisée par les instances politiques, d'une légitimation classiquement snob par l'affichage de goûts perçus comme élitistes à un positionnement paradoxal mettant en évidence un goût populaire. Le mouvement peut être assez précisément daté à la fin des années 1960 et au début des années 1970. Les hommes d'Etat cessent d'être surpris aux côtés de musiciens classiques, comme le faisaient leurs prédécesseurs. En France, le Président Pompidou et son épouse défendent l'art contemporain et la musique de Pierre Boulez. Son successeur Valéry Giscard d'Estaing jove de l'accordéon, «je cherche fortune » dès qu'il est ministre des Finances". II importe de comprendre ce qui pousse Bill Clinton à jover du saxophone, Vladimir Poutine à accueillir Mireille Mathieu comme le faisait Léonide Brejnev. Ce n'est pas l'abolition du snobisme «intellectuel » mais plutôt l'émergence d'une nouvelle forme de snobisme.

\section{De nouvelles formes de « distinction» et de légitimation}

Depuis les années 1990, le schéma de la « distinction», critique sociale du goût qui fonctionnait en musique depuis le livre fondateur de Pierre Bourdieub a été en partie complété ou remis en cause. Bernard Lahire insiste sur la coexistence dans les cultures individuelles de références savantes, légitimées comme étant de la haute culture et de formes plus «chaudes» de culture populaire?. On peut ainsi être snob dans un domaine culturel et non dans un autre. Si le snobisme a été l'expression d'un exclusivisme culturel se démarquant du goût commun et populaire en affectant le goût des élites auxquelles on n'appartient

\footnotetext{
5 http://www.ina.fr/video/1000 1920 //a-chamalieres-valery-giscard-d-estaing-a-l-accordeon-video.html

6 Pierre Bourdieu. A distinção: crítica social do julgamento. São Paulo: Edusp; Porto Alegre, RS: Zouk, 2007

7 Bernard Lahire, A cultura dos individuos, São Paulo, Artmed Editora, 2006
} 
pas vraiment, il semble aujourd'hui noyé dans un flot d'autres attitudes de démarcation culturelle contradictoires. La démarcation avec le vulgaire, l'agrégation mimétique aux strates supérieures se fait non pas sur un terrain référentiel unique mais dans une accumulation de systèmes culturels différents, voire contradictoires. Le terme qui renvoie à cette diversité culturelle est celui d'« omnivorisme » introduit dans la réflexion par Richard Peterson, terme qui peut être considéré comme un équivalent de l'idée d'éclectisme culturel généralisé. Richard Peterson décrit le passage du « snobisme intellectuel [qui] repose sur la glorification des arts et le dédain des divertissements populaires, [à un] capital culturel qui apparaît de plus en plus comme une aptitude à apprécier l'esthétisme différent d'une vaste gamme de formes culturelles variées qui englobent non seulement les arts, mais aussi tout un éventail d'expressions populaires et folkloriques $»^{8}$. La fin du snobisme correspond à une phase de globalisation culturelle: les élites peuvent se situer simultanément sur plusieurs échelles de légitimation culturelle, ne pas s'en tenir à une culture nationale, académique mais s'approprier les cultures populaires. Au temps du « vieux » snobisme, on pouvait avoir un capital culturel suffisant en se positionnant comme jazzophile ou fan de rock. Désormais, il convient de pouvoir se dire mélomane classique et amateur de musiques rares et méconnues. Loin d'être un signe de démocratisation, l'omnivorisme culturel implique des ressources plus abondantes et plus diverses pour «tenir son rang ». Comment en est-on arrivé à ce point? Posons l'hypothèse qu'avant d'exiger la diversification des ressources et des références le néo-snobisme omnivore naissant s'est concentré sur un renversement simple des valeurs.

\section{Le temps du kitsch : premier renversement de l'ordre du snobisme?}

\footnotetext{
8 Peterson Richard A., 2004. - «Le passage à des goûts omnivores : notions, faits et perspectives», Sociologie et sociétés, 36, 1, p. 147 . Voir également du même auteur «Understanding audience segmentation : from elite and mass to omnivore and univore », Poetics, 1992, pp. 243-258.
} 
Le renversement de la logique du snobisme s'est fait progressivement, avec des signes avant-coureurs qui apparaissent, par exemple, dans l'usage de la notion de kitsch. Notion que Norbert Elias parvient bien à historiciser?. La culture kitsch apparaît quand les sociétés doutent de la légitimité de leurs échelles de valeur esthétique. Le kitsch n'est pas une catégorie essentielle atemporelle mais une fausse catégorie esthétique produite par une société qui doute de ses valeurs et de ses goûts dominants. L'idée d'un kitsch musical n'est pas évidente. Dès que l'on cherche une équivalence entre un style visuel et un style musical, on se heurte à un risque d'impressionnisme. Quelle serait par exemple la musique qui correspondrait au style sulpicien dans l'art religieux? Comment la même sensibilité se traduit par «correspondance»? On peut ainsi d'écouter un musicien dont la gloire en 1900 était immense, Romain Rolland le considérant, par exemple, comme un génie: Don Lorenzo Perosi (1872-1956), par exemple son Transitus Animae (1907). L'amateur de kitsch ne remet pas en cause l'échelle des valeurs esthétiques et de la légitimation sociale des arts, il avoue une forme d'audace en affirmant son goût pour un objet singulier perçu comme contraire aux normes esthétiques. Assumer une part de mauvais goût, être « à contre-courant » revient à une forme différente de snobisme. Ce n'est pas seulement le renversement romantique d'un Hugo affirmant que le laid est beau : "la muse moderne verra les choses d'un coup d'œil plus haut et plus large. Elle sentira que tout dans la création n'est pas humainement beau, que le laid y existe à côté du beau, le difforme près du gracieux, le grotesque au revers du sublime, le mal avec le bien, l'ombre avec la lumière $\gg^{10}$. C'est une recherche relevant du snobisme de la perle de la laideur. Si l'on comprend mal comment jusqu'au moment où Luchino Visconti a fait (re)découvrir sa 5 e Symphonie, Gustav Mahler pouvait, parfois, passer pour un compositeur « kitsch », il est plus facile

\footnotetext{
9 Norbert Elias, 1998, «The Kitsch Style and the Age of Kitsch'. Translation from German by Edmund Jephcott of 'Kitschstil und Kitschzeitalter» (1935), in Johan Goudsblom and Stephen Mennell (eds), The Norbert Elias Reader: A Biographical Selection (Oxford: Blackwell), pp. 26-31.

10 Victor Hugo, Préface de Cromwell (1827), http://wuw.lanouvellecompagnie.org/lnc/images/stories/flexicontent/images_documents/prface\%20de\%20cromwell\%20-\%20victor\%20 hugo.pdf
} 
de suivre la recherche paradoxale de la laideur sublimée du snobisme «kitsch » lorsqu'il s'agit d'une chanteuse comme Florence Foster Jenkins". On aurait donc un snobisme classique (celui du Bourgeois gentilhomme), un âge d'or du snobisme avec, par exemple, le wagnérisme, un snobisme des avant-gardes après la rupture schönbergienne, et à partir de l'ère du kitsch divers post-snobismes ou néo-snobismes renversés et «omnivores ». L'hypothèse implique que l'on revienne aux définitions pour être bien sûr de parler du même phénomène social.

\section{Revenir à des définitions historiques de ce qui est désigné comme snob à l'âge du snobisme}

Avant de parler d'élargissement ou de renversement des attitudes esthétiques et sociales normatives, il importe de revenir au premier snobisme. On l'aura compris, le snobisme musical ne peut être coupé du snobisme culturel «global», comme celui que décrit Vian dans sa chanson déjà citée: «j'suis snob» implique que je me définis dans mon goût pour «le camembert à la petite cuillère » ou «les films suédois ». II sera important de comparer le snobisme musical et le snobisme propre à d'autres secteurs culturels. En attendant l'achèvement de la thèse de doctorat de Julie Demange sur «l'émergence et développement du mouvement bédéphile français dans les années 1960-1970 ${ }^{12}$, on peut supposer le passage du discours normatif classique ("moi, je ne lis jamais de bandes dessinées ») à deux types de discours, l'un relevant d'un nouveau snobisme ( moi, je ne lis que des bandes dessinées ») et l'autre d'un « omnivorisme » relatif qui peut être distinct du snobisme («moi, je lis aussi des bandes dessinées »). Le premier snobisme, le snobisme classique, revient à chercher à s'intégrer à des milieux sociaux jugés supérieurs en affectant de partager le goût qui y domine. C'est donc sur un critère unique que se pratique un double mouvement d'agrégation, de mimétisme, fondé sur une imitation, plus ou moins intériorisée et sincère,

\footnotetext{
II https://www.youtube.com/watch?v=yfaRnWUlQ4Al

12 Sujet de la thèse de doctorat déposée à l'Université Paris-I sous la direction de Pascal Ory en novembre 2013.
} 
et d'autre part, une façon de se démarquer des goûts qui caractérisent les groupes sociaux jugés inférieurs, des goûts du commun. Dans le verbe "snober», on retient l'idée de démarcation, d'exclusion des gens qui ont une culture. C'est un verbe transitif, on snobe quelqu'un, on l'exclut, on le traite de haut, on le méprise ou on le marginalise pour ses goûts. Le snobisme exclut et feint l'adhésion, c'est dire qu'il implique une situation sociale d'insécurité, le risque d'être à tout moment découvert. II implique une activité constante de démonstration. Si Pierre Reverdy a pu dire " II n'y a pas d'amour, il n'y a que des preuves d'amour », on pourrait penser que le snobisme est un peu du même ordre, il n'y a que des preuves d'adhésion ou de dédain. C'est cette insécurité que décrit plaisamment Boris Vian : "c'est une vie de galérien ». II ne peut être question de snobisme si le conformisme esthétique ne rencontre pas d'adversaire. Les lignes de partage évolvent et se reformulent dès qu'un combat esthétique se précise: querelle des Anciens et des Modernes, «bataille d'Hernani » de 1830. Pour nous en tenir au domaine de la musique, l'histoire contemporaine est émaillée par des «batailles» accompagnant Berlioz, Wagner, la création du Sacre du Printemps en 1913, les concerts publics d'œuvres de Schönberg 13, la première audition de Déserts d'Edgar Varèse en $1954^{14}$ ont été l'occasion de scandales fondateurs. Mais où est le snobisme, sans doute dans chaque camp. L'émergence et l'auto proclamation d'une nouvelle avant-garde est l'occasion de l'expression contradictoire de snobismes ennemis qui consistent assez souvent à accuser l'autre camp de snobisme, le plus souvent en usant d'un discours violent et d'invectives ${ }^{15}$. On ne peut pas assimiler de façon univoque l'avant-garde au camp du snobisme. II existe un snobisme de l'authenticité baroque, de l'attachement exclusif à une esthétique, à une hauteur du diapason, à un interprète qui n'est pas nécessaire-

13 Esteban Buch, Le cas Schönberg. Naissance de l'avant-garde musicale Collection Bibliothèque des Idées, Gallimard, 2006, 368 p.

14 Julien Mathieu, «Un mythe fondateur de la musique contemporaine : le « scandale » provoqué en 1954 par la création de Déserts d'Edgar Varèse », Revue d'histoire moderne et contemporaine 1/ 2004 (no5 1-1), p. 129-152.

URL: www.cairn.info/revue-d-histoire-moderne-et-contemporaine-2004-1-page-129.htm.

15 Enregistrement de la création mondiale de Déserts de Varèse: https://www.youtube.

com/watch? $v=$ _ihrJ2 $-8 \times a 0$ 
ment d'avant-garde. II peut y avoir un snobisme d'arrière-garde. Mais il faut qu'il y ait une opposition entre générations, entre esthétiques, entre strates des élites pour que le discours du snobisme prospère. Le fait qu'il y ait aussi un snobisme d'avant-garde indique bien que la volonté d'agréger la classe supérieure (les bourgeois adoptant un mode de vie aristocratique) n'est pas nécessairement au cœur du snobisme. II ne s'agit pas pour un snob d'avant-garde de s'intégrer à un groupe étroit et fermé en imitant ses goûts. Les vieilles aristocraties n'ont pas besoin d'avant-garde. L'avant-garde serait plutôt une stratégie offensive d'élites nouvelles, mais elle se sépare de la masse dans un schéma conflictuel. Un subtil équilibre doit s'établir entre ce qui permet de ressembler aux nobles et ce qui permet de se positionner comme un aristocrate du goût méprisant le sens commun. II faut être à la fois original et conforme à la culture légitimée par les élites. Cela correspond assez bien à la dialectique de la mode décrite par Georg Simmel'b.

Entre conformisme et originalité, le discours snob consiste à proclamer l'exclusivité de ressources culturelles, inaccessibles aux autres, au commun et différente de celles qu'ont accumulées les anciennes élites. L'expression de Sartre sur le jazz ("c'est comme les bananes, cela doit se consommer sur place ») est significative d'une des constantes du snobisme musical: "je sais que c'est mieux ailleurs, j'ai accès à quelque chose d'une beauté plus rare, moins évidente, plus cachée que mes ressources me permettent de connaître et de percevoir ». Le mélomane snob se pose en médiateur entre la société ignare et une réalité musicale méconnue à laquelle il est un des rares privilégiés à avoir accès. II faut aller en Amérique écouter du jazz, avoir été à Bayreuth ou à Milan. Ce qui conduit Frédéric Rouvillois à considérer que «le cosmopolitisme, ce que l'on pourrait nommer la «xénolâtrie », c'est-à-dire l'amour systématique de ce qui est étranger ou de ce qui vient de l'étranger entretient avec ce snobisme des relations complexes ${ }^{17}$. Ce type d'analyse est en partie lié à l'idée que les élites adaptées, à l'ère de la mondialisation, ne sauraient pas comprendre ce que les masses populaires ne

\footnotetext{
${ }_{16}$ Georg Simmel, Philosophie de la mode, Allia, 2013, 64 p.

17 Frédéric Rouvillois Histoire du snobisme, Flammarion 2008, p. 101.
} 
peuvent pas accepter dans le cosmopolitisme. II est difficile de ne pas exprimer une certaine xénophobie lorsque l'on fustige le cosmopolitisme des élites. L'anti snobisme est, on l'a compris, une forme de snobisme, il peut aussi dériver vers des formes d'enfermement identitaire.

Dans le snobisme et l'anti snobisme musical, les questions de positionnement social et théorique s'accompagnent d'une pratique active d'appropriation. Le plaisir de l'écoute peut être bien présent. II est de bon ton de pouvoir décréter que telle interprétation d'une œuvre musicale est «la seule ». Mais pour y parvenir, il faut parfois écouter d'autres versions, d'autres avis pour se conformer à ce que l'on perçoit comme le plus distingué. En France, une émission hebdomadaire célèbre était consacrée de 1946 à 1983 à la comparaison de différentes interprétations d'une même œuvre ${ }^{18}$. Antoine Coléa, Jacques Bourgeois et Jean Roy s'affrontaient en mesurant les mérites comparés de tel chef ou de tel orchestre pour finir par définir les interprétations de référence. Mais même lorsque la porte est ouverte à la reconnaissance d'un certain pluralisme culturel et musical, à l'idée de confronter des sensibilités différentes toutes aussi légitimes les unes que les autres, le snobisme consiste à poser l'existence d'une communauté érudite et mélomane qui dispose d'un capital culturel comparable et de références communes. Un producteur d'émissions télévisées musicales françaises, organiste, Bernard Gavoty présente une prestigieuse interprétation de la Sonate à Kreutzer, il ajoute, de façon significative du «comme de bien entendu » de Beethoven « une précision superflue, j'en suis sûr » ${ }^{19}$, préférant ignorer Tolstoï... Le snobisme se trouve ainsi à tout moment menacé dans une situation de tension, avec l'émergence d'autres critères, il se trouve ainsi dans un phénomène historique permanent de construction et de reconstruction.

\footnotetext{
18 http://www.ina.fr/video/CPF86625879

19 Didier Francfort, «La musique classique à la télévision française des années 1950 aux années $1990 »$, in Le Temps des Médias. N²2, printemps-été 2014, p. 110.
} 


\section{Du «Bourgeois gentilhomme » au pèlerin de Bayreuth. Construction et déconstruction du snobisme musical}

L'histoire du snobisme musical est ainsi marquée par des temps forts d'affrontements. La dénonciation avant que le mot existe du snobisme de Monsieur Jourdain correspond à une nostalgie d'un ordre social menacé par la progression des bourgeois, par ce que l'on appelle les «savonnettes à vilains». Puis, le mélange entre conformisme et originalité dans la légitimation culturelle des goûts musicaux s'épanouit lors d'oppositions entre goûts rivaux, lors de la querelle des bouffons au milieu du XVIIle siècle, on affirme son goût pour ou contre l'italianisation de l'opéra français en se différenciant du camp adverse en affichant une meilleure perception de ce qu'est la musicalité : Rameau contre Rousseau. Le dédain affiché pour les bourgeois dans le camp des romantiques hostiles aux classiques semble rattacher davantage le snobisme au romantisme qu'au classicisme.

S'il y a un phénomène musical qui coïncide avec la généralisation du terme de snob dans la seconde moitié du XIXe siècle, c'est le wagnérisme. En être ou pas, aller à Bayreuth, lire des revues, reconnaître les leitmotivs. Tout cela correspond bien aux codes du snobisme, mêlant conformisme de groupe, d'élite et sens de l'originalité dans la mode. Le salon de Madame Verdurin chez Proust est marqué par l'émotion wagnérienne. C'est bien à ce moment que se fixe un modèle de snobisme musical. Lorsque dans son exil argentin, Witold Combrowicz va au théâtre Colon, il note dans son journal son passage au foyer : «Je me croyais au milieu des héros de Proust où personne ne va au concert pour écouter, mais uniquement pour magnifier la réunion de sa présence, où les grandes dames s'épinglent du Wagner dans les cheveux [...] $»^{20}$. Wagnériens et antiwagnériens ont créé à la fin du XIXe siècle un terrain commun de légitimation culturelle de la musique, mêlant mélomanie et mondanité. Dans le livre de Pierre Veber, Chez les snobs paru en 1896, les snobs fréquentent l'opéra pour essayer d'intégrer les élites et de se

20 Witold Combrowicz, Journal, tome I: 1953-1958, Trad. du polonais par Dominique Autrand, Christophe Jezewski et Allan Kosko, Collection Folio ( $\left.n^{\circ} 2767\right)$, Gallimard, p. 78. 
proclamer à l'avant-garde. Ils sont définis comme ceux « qui portent la dernière mode, qui veulent tout comprendre, ou paraître tout comprendre, et qui n'estiment que le rare et le précieux et tombent ainsi dans l'extravagant $»^{2 !}$.

Ce qui est vrai des wagnériens demeure lorsque la musique de Debussy prétend rompre avec le wagnérisme. II y a une façon de se pâmer avec exagération qui est un geste de snobisme. Jean Lorrain dresse un portrait acide de ce groupe de Debussystes qu'il surnomme non sans allusions sexuelles les «Pelléastres»:

«Convulsés d'admiration aux pizzicati soleilleux du petit chef-d'œuvre qu'est l'Après-midi d'un faune, ils ont décrété l'obligation de se pâmer aux dissonances voulues des longs récitatifs de Pelléas. L'énervement de ces accords prolongés et de ces interminables débuts d'une phrase cent fois annoncée ; cette titillation jouisseuse, exaspérante et à la fin cruelle, imposée à l'oreille de l'auditoire par la montée, cent fois interrompue, d'un thème qui n'aboutit pas; toute cette œuvre de Limbes et de petites secousses, artiste, oh combien! quintessenciée... tu parles ! et détraquante... tu l'imagines! devait réunir les suffrages d'un public de snobs et de poseurs. Grâce à ces messieurs et à ces dames, M. Claude Debussy devenait le chef d'une religion nouvelle et ce fut, dans la Salle Favart, pendant chaque représentation de Pelléas, une atmosphère de sanctuaire.

On ne vint plus là qu'avec des mines de componction, des clins d'yeux complices et des regards entendus. Après les préludes écoutés dans un religieux silence, ce furent, dans les couloirs, des saluts d'initiés, le doigt sur les lèvres, et d'étranges poignées de mains hâtivement échangées dans le clair-obscur des loges, des faces de crucifiés et des prunelles d'au-delà.

La musique est la dernière religion de ce siècle sans foi. Les auditions de Tristan et de Parsifal entassent, au Châtelet,

${ }^{21}$ Pierre Veber, op. cit. p. 9. 
dans les places supérieures, une population ardente et figée d'hypnose en tout point pareille à celle des premiers chrétiens assemblés dans les Catacombes. Mais, au moins, les adeptes de Wagner sont sincères: ils se recrutent dans toutes les classes sociales et l'humilité des vêtements, la laideur parfois sublime des visages contractés, témoignent de la ferveur et de la violence de leur foi. La religion de M. Claude Debussy a plus d'élégance; ses néophytes peuplent surtout les fauteuils d'orchestre et les premières loges, les stalles d'orchestre aussi, parfois. $»^{22}$.

Le phénomène de succession des modes auxquels les snobs sont condamnés apparaît clairement. Quand un goût se généralise, quand une mode se démocratise, il faut en trouver une autre. De telles conversions sont perçues extérieurement comme des formes d'affectation non sincères. Faute de savoir ce qu'est la réalité du goût du présumé snob, les autres, devenus spectateurs de sa passion, le catégorisent comme snob en refusant de considérer que sa passion est sincère. Proust a bien noté cela à propos de Legrandin. "Et certes cela ne veut pas dire que M.Legrandin ne fût pas sincère quand il tonnait contre les snobs. II ne pouvait pas savoir, au moins par lui-même qu'il le fût, puisque nous ne connaissons jamais que les passions des autres, et que ce que nous arrivons à savoir des nôtres, ce n'est que d'eux que nous avons pu l'apprendre $»^{23}$. Legrandin voit l'« attrait de l'esprit et de la vertu qu'ignorent les infâmes snobs. Seuls les autres savaient qu'il en était un; car, grâce à l'incapacité où ils étaient de comprendre le travail intermédiaire de son imagination, ils voyaient en face l'autre activité mondaine de Legrandin et sa cause première ». Le snobisme finit ainsi par plonger l'individu dans un doute sur son propre goût: le dédain social, la volonté d'intégrer les cercles les plus élitistes s'accompagnent d'un questionnement sur le fait que subsiste en soi-même des zones d'ombres et des goûts d'une grande banalité.

\footnotetext{
22 Jean Lorrain, Les Pélléastres, Paris, 1910, pp. 24-25.

23 Marcel Proust, op. cit. p. 129.
} 


\section{Affectation, mensonge et haine de soi}

Bernard Lahire a mis en évidence une certaine articulation entre le phénomène social du snobisme et de la distinction et la façon dont l'opposition entre noble et populaire, entre légitime et illégitime, jove dans l'individu lui-même, le poussant à « détester la part populaire de soi »: l'opposition repose sur des « différences entre classes certes - avec le «peuple » comme figure repoussoir dont il faut à tout prix se démarquer - mais aussi des différences de soi à soi (entre un état actuel de soi et un état antérieur de soi ou entre états parallèles de soi), ces dernières pouvant donner lieu à des luttes de soi (légitime) contre soi (peu légitime) »24. II y a peut-être une spécificité musicale dans cette fission du sujet esthétique. Je peux masquer mon goût pour les cartes postales de couchers de soleils, je peux ne pas dire que je continue à voir tel fevilleton télévisé et ne pas être obsédé par ce fevilleton dès que le générique de fin défile sur l'écran. La musique populaire résonne en moi plus longtemps que je ne le voudrais. Elle me hante, m'obsède. Le terme allemand d'Ohrwurm (ver d'oreille) désigne bien ces airs entêtants dont on ne parvient pas à se débarrasser ${ }^{25}$. Ce sont des tubes commerciaux mais cela peut être un air d'enfance, une phrase musicale.

II se peut que cette part inévitable de goût musical illégitime conduise au déploiement d'une agressivité contre le « goût des autres». Dès lors que je n'aime pas un style de musique, je me trouve prêt à accuser ceux qui disent l'aimer d'insincérité et donc de snobisme. L'accusation est assez systématique dès lors qu'il s'agit de musiques contemporaines. Dans l'Histoire du snobisme de Frédéric Rouvillois (2008), l'index thématique, en matière de musique, ne renvoie systématiquement, de façon significative, qu'à la musique contemporaine. Le livre s'en prend particulièrement à John Cage et à ses 4'33" en assimilant sa démarche à un canular à une plaisanterie.

\footnotetext{
24 Bernard Lahire, «Distinctions culturelles et lutte de soi contre soi : " détester la part populaire de soi »», in Hermes, Paris, 2005, p. 138.

25 Peter Szendy, Tubes : la philosophie dans le juke-box. , Paris, éditions de Minuit, 2008, $94 \mathrm{p}$.
} 
Selon Frédéric Rouvillois, ce qui se passe dans le snobisme est le contraire de ce que Marx décrivait dans le 18 Brumaire de Lovis Napoléon Bonaparte, I'histoire se répète mais la seconde fois c'est sous forme de farce grotesque. Là, dans la musique contemporaine, le canular, la blague se répètent en étant prise au sérieux, "suscitant des admirateurs inconditionnels et des exégètes ». II faut dire que la vision de Rouvillois exclut une bonne partie des musiques du XXe siècle, du Sacre du Printemps à Schoenberg, qui perçues comme réservées aux snobs ont fini par acquérir un statut de classiques contemporains. L'accusation de snobisme, comme le remarque Proust à propos de Legrandin, est une négation de la sincérité du goût mais aussi une négation du «travail intermédiaire » de l' «imagination » du mélomane suspect de snobisme. Si l'on reprend l'idée des snobismes croisés, ou plutôt des accusations croisées de snobisme, on peut partir de l'hypothèse que le principal acte de snobisme consiste à dénoncer le snobisme des autres, qui ne peuvent quand même pas aimer sincèrement l'épouvantable musique quils prétendent défendre. On ne peut aimer sincèrement cela, c'est donc que l'on dit aimer par snobisme pour se positionner dans les élites. Cela conduit par exemple Adorno à forger l'idée du caractère fétiche dans la musique, seule valeur marchande de musiques sans intérêts conduisant à des écoutes régressives. Adorno serait prêt à taxer de snobisme un intellectuel qui dirait aimer le jazz. Inversement, un jazzophile serait prêt à taxer de snobisme un adornien qui professerait une admiration exclusive pour Webern. Dans les deux cas, l'accusation est de défendre une musique non pour sa valeur mais pour son usage et d'en faire un fétiche, que cela soit une pièce de canular élevée au rang de chef d'œuvre par des snobs prétentieux ou une piètre musique de danse célébrée comme un chef d'œuvre malgré son évidente pauvreté par un ignare sûr de son jugement. Dans les deux cas, l'accusation de snobisme revient à dire que le sujet n'a pas pris le temps du «travail intermédiaire » nécessaire à une juste perception d'une œuvre.

Le snobisme apparaît ainsi comme un court-circuit dans l'élaboration du goût musical. Le snob est alors à l'opposé de l'érudit, il est en mesure de juger, de classer sans s'appesantir. Dans Chez les snobs 
(1896), Pierre Veber définissait les snobs comme des gens «qui portent la dernière mode, qui veulent tout comprendre, ou paraître tout comprendre, et qui n'estiment que le rare et le précieux et tombent ainsi dans l'extravagant $»^{26}$. C'est l'immensité de l'ambition de ce «tout» qui implique une rapidité constante dans le jugement esthétique et dans la mise en évidence de la parfaite connaissance de toutes les références nécessaires. Ainsi, l'« inédit», «le rare et le précieux», «l'extravagant», l' «étrangeté » et le «cosmopolitisme » sont recherchés par les snobs essentiellement parce qu'ils leur permettent de démontrer leur maîtrise des circuits de diffusion et de légitimation qu'ils ont les moyens de court-circuiter pour arriver avant les autres à ce qui est qualifiant. Ainsi si la comparaison des interprétations des classiques est un bel exercice de snobisme pratique, il en est un autre qui repose sur la multiplication des citations à reconnaître. «Je sais ce que c'est » constitue le credo du snobisme musical.

Aucune musique n'échappe à un recours à des formes spécifiques reconnaissables ou à des citations qui peuvent fonctionner comme des marqueurs pour snobs. Un exemple de ces citations, une version par Charles Mingus d'All the things you are, introduit par... du Rachmaninov27. Les joutes musicales parodiques de Gérard Hoffnung sont à bien des égards des jeux pour initiés, jubilant de reconnaître plus vite que leur voisin la citation qui se découvre à leurs oreilles ${ }^{28}$. Cette rapidité dans la reconnaissance d'une musique peut conduire à chercher dans le terme même de « reconnaissance » une clef du snobisme et pour cela à lire Paul Ricœur29. Les multiples sens en français de l'idée de reconnaissance sont au cœur de la définition, par Paul Ricœur, des «parcours de reconnaissance», je reconnais les autres, je classe les objets, je me sens estimé par les autres, j'exprime ma gratitude. Tout

\footnotetext{
26 Pierre Veber, op.cit. p. 9.

27 https://www.youtube.com/watch? v=kCAhdURwa7| consulté le 30 novembre 2014.

28 Ecouter, par exemple le «Concerto Popolare »ou « concerto pour en finir avec tous les concertos » attribué à un certain Franz Reizenstein, enregistré en public en 1956: https:// wuw.youtube.com/watch? V=PVC IAkIJh68 consulté le ler décembre 2014.

29 Paul Riccur, Parcours de la reconnaissance. Trois études. Collection Folio essais ( $n^{\circ}$ 459), Gallimard, 2005, 448 p.
} 
cela procède de la reconnaissance. Le snobisme musical (« ambition sociale » comme le dit Proust) consiste à chercher la reconnaissance sociale en montrant que l'on est capable très vite de reconnaître et de juger toute forme de musique, que l'on maîtrise les codes esthétiques et sociaux, que l'on sait tout, en définitive. Le snobisme musical est donc une forme de raccourci entre le moment d'une reconnaissance active je reconnais un objet - à une reconnaissance passive correspondant à mon besoin d'être reconnu, sinon aimé. Le personnage de Sebastyan dans le film de Lubitsch That uncertain feeling (1941) résume assez bien le snobisme musical, fait social de démarcation des braves bourgeois assureurs et marchands. II juge Wagner en une demi-phrase, impose son goût comme seul légitime et parvient ainsi à séduire ${ }^{30}$.

Cette réflexion sur le snobisme musical, capacité à se positionner dans les élites à partir d'un capital culturel à la fois original et conforme aux codes particuliers réels ou supposés de la frange la plus étroite des élites sociales s'achève sur une série de constats dont la simplicité pourra décevoir. On est toujours le snob de quelqu'un et le jeu de reconnaissance et de différenciation peut facilement s'inverser comme l'a montré Marcel Proust. En ce sens, nous sommes tous snobs, nous ne sommes pas à l'abri d'une volonté de nous hausser à une forme de reconnaissance supérieure en brusquant nos goûts pour les rendre plus distingués ou paradoxaux, quitte à risquer de tomber dans une haine schizophrénique de la part populaire et «facile » de nos goûts musicaux.

La fin de ce phénomène est significative, en période de globalisation culturelle, d'un autre mode de distribution du capital culturel. L'omnivorisme culturel consiste à valoriser la diversité des références, des codes et donc de manifester une capacité à maîtriser l'immense corpus musical dématérialisé qui circule, le critère de distinction ne se fonde plus sur un classement binaire «beau et laid» mais sur l'idée d'un intérêt qui crée un désir d'appropriation. Si le snob a été remplacé par l'omnivore culturel des élites, restent d'immenses groupes sociaux qui n'ont

30 Voir en particulier la soirée « hongroise » des minutes 31 à 37 :

https://www.youtube.com/watch?v=msLUcn 4 aqKg 
pas accès à l'omnivorisme et sont condamnés à un régime unique, végétarien ou trop carné. Les omnivores deviennent des ogres capables d'ingérer un monde musical de plus en plus diversifié, alors que les autres (ceux qui n'ont pas accès à la diversité) vivent dans un monde sonore qui se rétrécit et se renferme. Le discours politique identitaire des nouvelles droites «populaires » européennes se nourrit de cette frustration. Pour ces exclus de l'omnivorisme, les nantis, les classes aisés restent, dans leur omnivorisme, des snobs ou des «intellos de gauche » coupés des réalités quotidiennes. Eux votent à présent pour l'extrême droite. 